ARTICLE

\title{
A droplet microfluidic platform for high-throughput photochemical reaction discovery
}

\author{
Alexandra C. Sun ${ }^{1,2}$, Daniel J. Steyer ${ }^{1,2}$, Anthony R. Allen (10 ${ }^{1}$, Emory M. Payne (i) ${ }^{1}$, Robert T. Kennedy (D) ${ }^{1 凶} \&$
} Corey R. J. Stephenson (D) ${ }^{1 \times}$

The implementation of continuous flow technology is critical towards enhancing the application of photochemical reactions for industrial process development. However, there are significant time and resource constraints associated with translating discovery scale vialbased batch reactions to continuous flow scale-up conditions. Herein we report the development of a droplet microfluidic platform, which enables high-throughput reaction discovery in flow to generate pharmaceutically relevant compound libraries. This platform allows for enhanced material efficiency, as reactions can be performed on picomole scale. Furthermore, high-throughput data collection via on-line ESI mass spectrometry facilitates the rapid analysis of individual, nanoliter-sized reaction droplets at acquisition rates of 0.3 samples $/ \mathrm{s}$. We envision this high-throughput screening platform to expand upon the robust capabilities and impact of photochemical reactions in drug discovery and development.

\footnotetext{
${ }^{1}$ Department of Chemistry, University of Michigan, Ann Arbor, MI, USA. ${ }^{2}$ These authors contributed equally: Alexandra C. Sun, Daniel J. Steyer.

凶email: rtkenn@umich.edu; crjsteph@umich.edu
} 
igh-throughput experimentation (HTE) techniques hold the potential to revolutionize modern catalysis and reaction discovery by enabling the exploration of myriad reaction conditions in a time and resource-efficient manner ${ }^{1-6}$. In recent years, efforts have been directed towards the development of mass spectrometry-based (MS) HTE systems for the automated processing of Pd-based cross-coupling reactions on nanomole scale, in both batch and continuous flow settings ${ }^{7-10}$. In particular, the development of flow-based HTE platforms enables the direct optimization of continuous flow reactions in a materialefficient and data-rich manner. Researchers at Pfizer have developed a modular, automated system to enable nanomole scale reaction discovery in-flow at a throughput of over 1500 samples/ day $(0.02 \text { samples/s })^{9}$. While reported HTE flow systems have been successfully applied to non-photochemical reactions, demand remains for the development of HTE platforms for screening photochemical reactions in continuous flow.

Among the repertoire of modern catalytic methods, photoredox catalysis has enabled otherwise infeasible bond disconnections and aided sustainability efforts in industrial synthesis $^{11}$. The widespread implementation of photoredox catalysis renders the development and adaptation of flow technology to be broadly impactful for reaction scale-up ${ }^{12,13}$. The greater surface area-to-volume ratio accessible in-flow results in increased photon flux, which can lead to reaction acceleration ${ }^{12}$. Translating discovery-scale batch conditions to pilot-scale flow operations often necessitates significant resource consumption. Additionally, the process of re-optimization for flow conditions can prove laborious and cost-inefficient ${ }^{14}$. While commercially available systems have been designed for photochemical highthroughput batch (i.e., microvial-based) experimentation ${ }^{15}$, there is comparatively limited capability for performing flow-based screening of photochemical reactions. Those flow-based screening systems that have been reported lack the throughput of their batch counterparts ${ }^{16-18}$. With the end goal of developing scalable photochemical flow reactions, we aimed to build an HTE system to enable reaction discovery in-flow.

We have identified droplet microfluidics as a platform that may enable continuous flow-based reaction discovery. Segmentation of samples with an immiscible phase enables the simultaneous handling of numerous samples over extended periods of time ${ }^{19-21}$. From a material consumption standpoint, droplet microfluidics screens are typically performed at nanoliter to femtoliter scale, which translates to a reduction in starting material usage by three to eight orders of magnitude relative to a traditional multiwell plate-based screen. Droplet microfluidics is also well suited for photocatalytic reactions, as the micrometer dimension of the reaction vessel allows for high photon flux through the reaction channel in an analogous manner to the narrow tubing employed in-flow reactors ${ }^{22-24}$. Here, we report advances towards a droplet microfluidic/MS platform to enable picomole-scale discovery of visible light-driven reactions and provide robust translatability to millimole flow scale-up processes. In particular, the design of a flow-based screening platform that interfaces with pre-plated compound libraries would allow for facile integration into existing pharmaceutical HTE workflows and infrastructures. We foresee the synergistic combination of droplet microfluidics, MS, and photoredox catalysis as an enabling platform for accelerating pharmaceutical discovery and development, with a concerted emphasis on time and material efficiency.

\section{Results}

ESI-MS analysis of in-droplet reactions. In this work, we aimed to develop a system for irradiating droplet samples, followed by in-line dilution and subsequent electrospray ionization-mass spectrometry (ESI-MS) analysis (Fig. 1). Droplet samples $(5-10 \mathrm{~nL})$ were generated from a standard 384 or 1536 microwell plate using previously reported methods ${ }^{25-28}$, enabling the facile translation of a wide variety of reaction conditions into droplet format. In our preliminary studies, we constructed an easily assembled, low-footprint photoreactor (see Supplementary Information for details). Following droplet irradiation, simultaneous dilution and ESI-MS analysis of each individual droplet sample was performed using a sheath sprayer, which enables in-line dilution of samples as they are pumped into the ESI source ${ }^{25}$. Dilution of droplets served to both quench the reaction and facilitate MS analysis, as the analysis of high concentration $(>1$ $\mathrm{mM}$ ) analytes can lead to saturation of MS signal and contamination of the MS source. In our preliminary studies, we chose to employ a
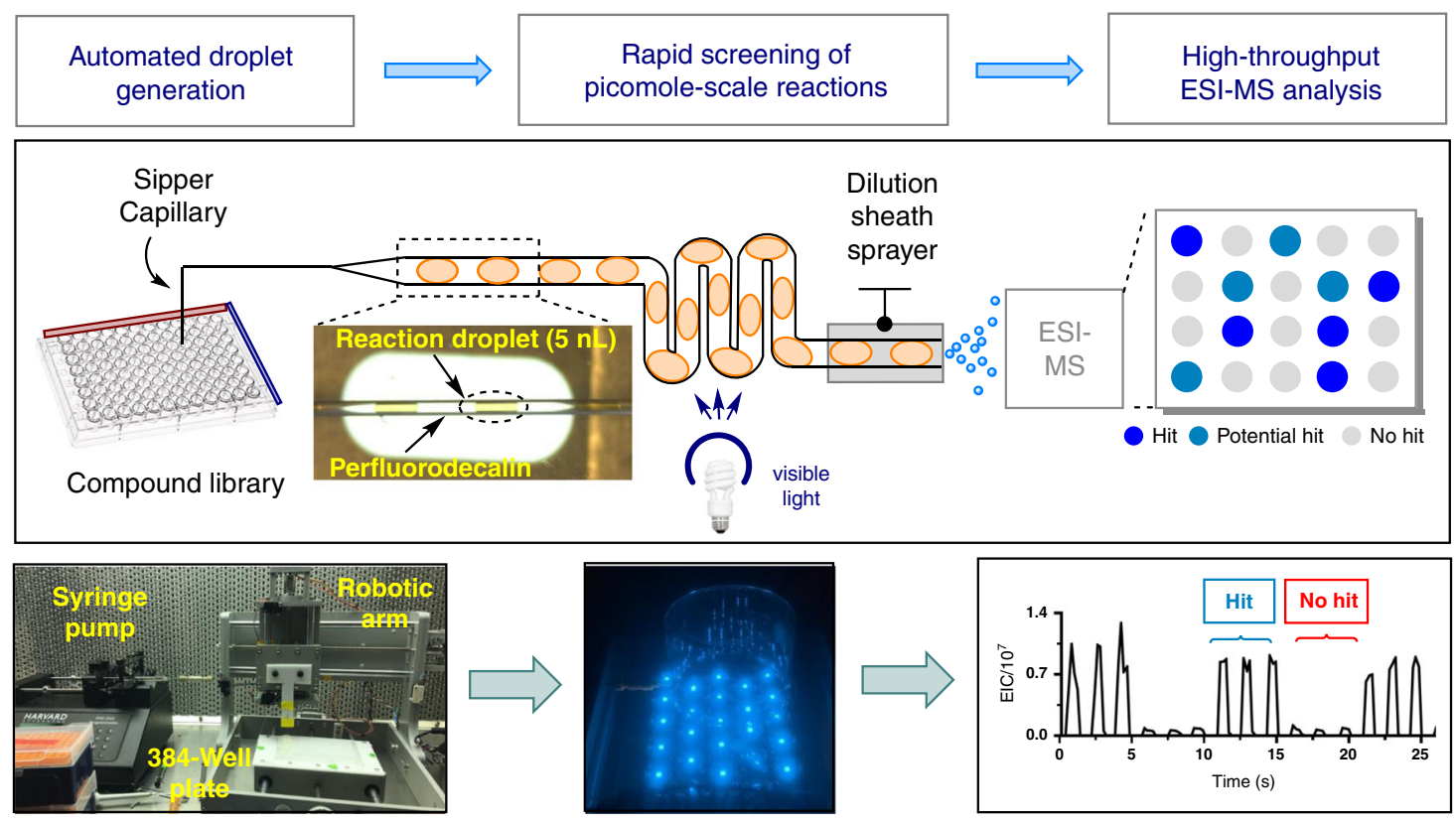

Fig. 1 Development of a photochemical droplet microfluidics platform. EIC extracted ion chromatogram, ESI-MS electrospray ionization-mass spectrometry. 


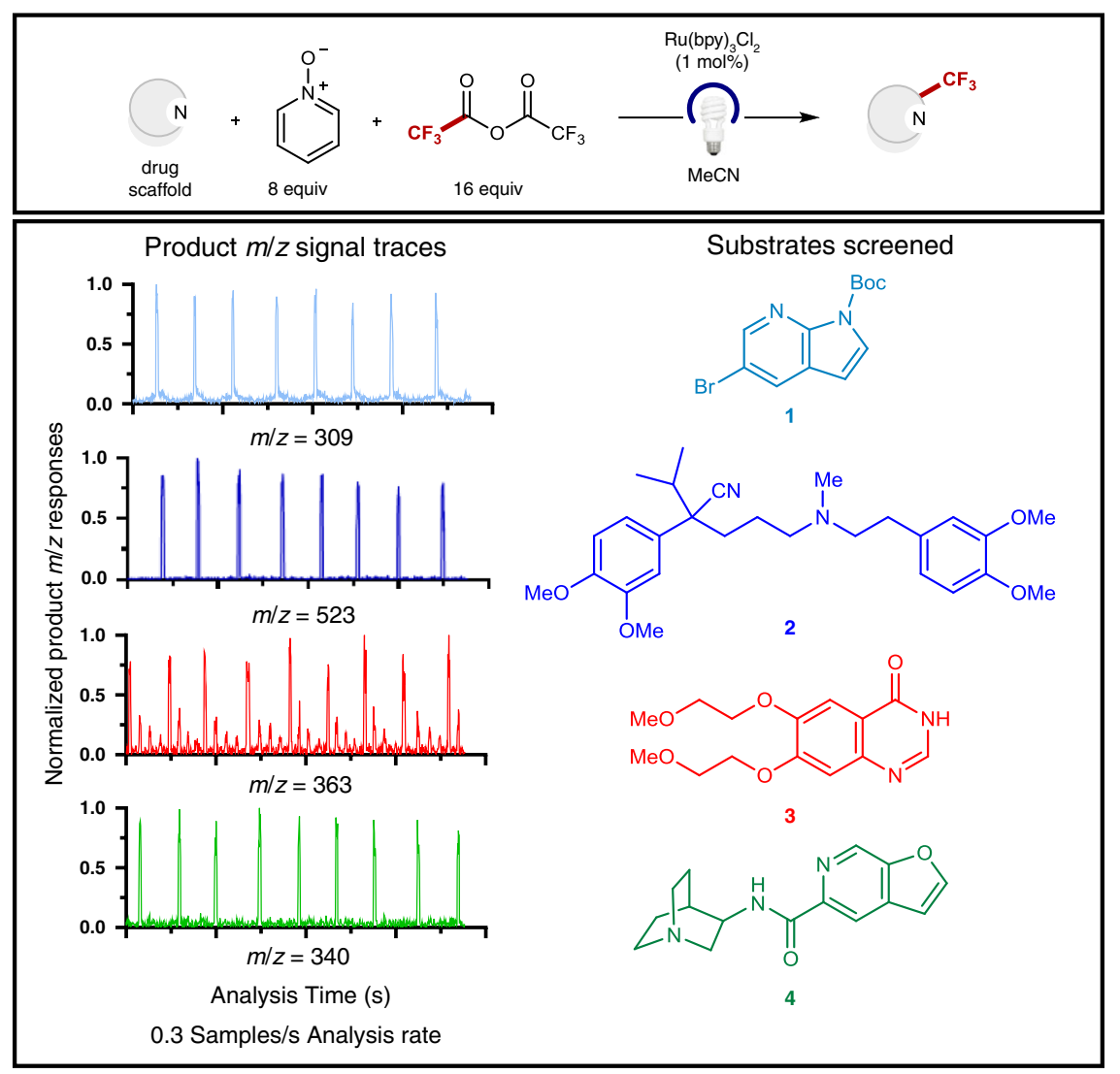

Fig. 2 ESI-MS analysis of in-droplet trifluoromethylation reactions. $\mathrm{MH}^{+}$molecular ions were monitored for all trifluoromethylated products except for 1 , which was prominently observed as a $\mathrm{m} / z=309$ fragment. $\mathrm{Ru}(\mathrm{bpy}){ }_{3} \mathrm{Cl}_{2}$ tris(bipyridine)ruthenium(II) chloride.

radical trifluoromethylation developed by the Stephenson group ${ }^{29}$ as a model reactive system for the late-stage functionalization of complex pharmaceutical intermediates. To demonstrate the general operation of our system, we performed in-droplet trifluoromethylation of four distinct substrates (Fig. 2,1-4), three of which were either approved therapeutics or drug candidates provided by a commercial library from Pfizer ${ }^{30}$. Reaction droplets $(4 \mathrm{~nL})$, segmented by a perfluorodecalin (PFD) carrier phase $(8 \mathrm{~nL})$, were formed in a 100 $\mu \mathrm{m}$ internal diameter (i.d.) PFA tubing. Droplet reactions containing different substrates were generated in a consecutive manner. After a $10 \mathrm{~min}$ irradiation, droplets flowed at $500 \mathrm{~nL} / \mathrm{min}$ into the sheath sprayer for subsequent dilution. ESI-MS analysis of droplet samples was performed at $0.3 \mathrm{samples} / \mathrm{s}$. As anticipated, prominent product $\mathrm{m} / \mathrm{z}$ signals were observed iteratively throughout the four traces in the expected alternating pattern. These results validate the capability to perform rapid MS analysis while maintaining the identity of individual samples. Notably, the analytes were not subject to Taylor dispersion as in a conventional plug flow reactor ${ }^{12}$. For the reactions in Fig. 2, we also observed reproducible results in both our droplet-todroplet analysis and in-droplet chemistries. (Supplementary Fig. 3). Droplet ESI-MS measurement of reaction turnover yielded RSD values ranging from 1-19\% across all four reactions, both for reactions run prior to droplet generation and for reactions performed inside of droplets. In this manner, we established proof-of-concept for the successful performance and ESI-MS analysis of in-droplet organic synthesis reactions.

In-droplet reaction discovery. In the next phase of our studies, we set out to investigate in-droplet photoredox flow reactions. To perform residence time optimization, we designed an oscillatory flow system to enable extended irradiation of reaction droplets (Supplementary Fig. 5). Oscillation was induced by alternating a syringe pump between withdrawal and infusion modes while maintaining a constant flow rate of $200 \mathrm{~nL} / \mathrm{min}$. Initial validation of our oscillatory system was performed using a visible lightdriven alkene aminoarylation reaction ${ }^{31}$. Successful product formation was observed by ESI-MS analysis, with droplet identities maintained after a $1 \mathrm{~h}$ residence time. Notably, while state-of-theart oscillatory flow systems have been limited to a single reaction plug during each incubation period, this setup could potentially accommodate $>100 \times$ more samples per incubation period ${ }^{19,32}$.

After observing success with our droplet flow reactor, we sought to perform HTE reaction discovery to generate a library of alkene aminoarylation products. We aimed to screen an extensive library of sulfonylacetamides and alkenes on a picomole scale to furnish a wealth of reactivity data generated from each substrate combination. Ten sulfonylacetamides and ten alkenes were selected for our screen (500 pmol scale), resulting in the potential generation of 100 distinct product combinations (Fig. 3). For these studies, droplet reactions were irradiated using a custombuilt Cree LED array photoreactor, in order to maximize photon flux $^{27}$. Our oscillatory flow setup was employed to maintain a residence time of $30 \mathrm{~min}$ at a flow rate of $200 \mathrm{~nL} / \mathrm{min}$ during reaction irradiation (100-200 reaction droplets per incubation period). ESI-MS analysis was performed at a throughput of 0.3 samples/s (350 total samples over $19 \mathrm{~min}$ ). ESI-MS results suggested the identification of 37 hit conditions, for which significant product $\mathrm{m} / z$ signals were observed. The employment of trans-anethole yielded product formation across the entire scope of sulfonylacetamide substrates, in-line with reported studies in batch ${ }^{31}$. Notably, pharmaceutically relevant sulfonylacetamides containing heteroarenes $(6,10)$ also yielded several hit responses in our droplet screen. Furthermore, this data suggests that electron deficient sulfonylacetamides generally gave rise to 
a
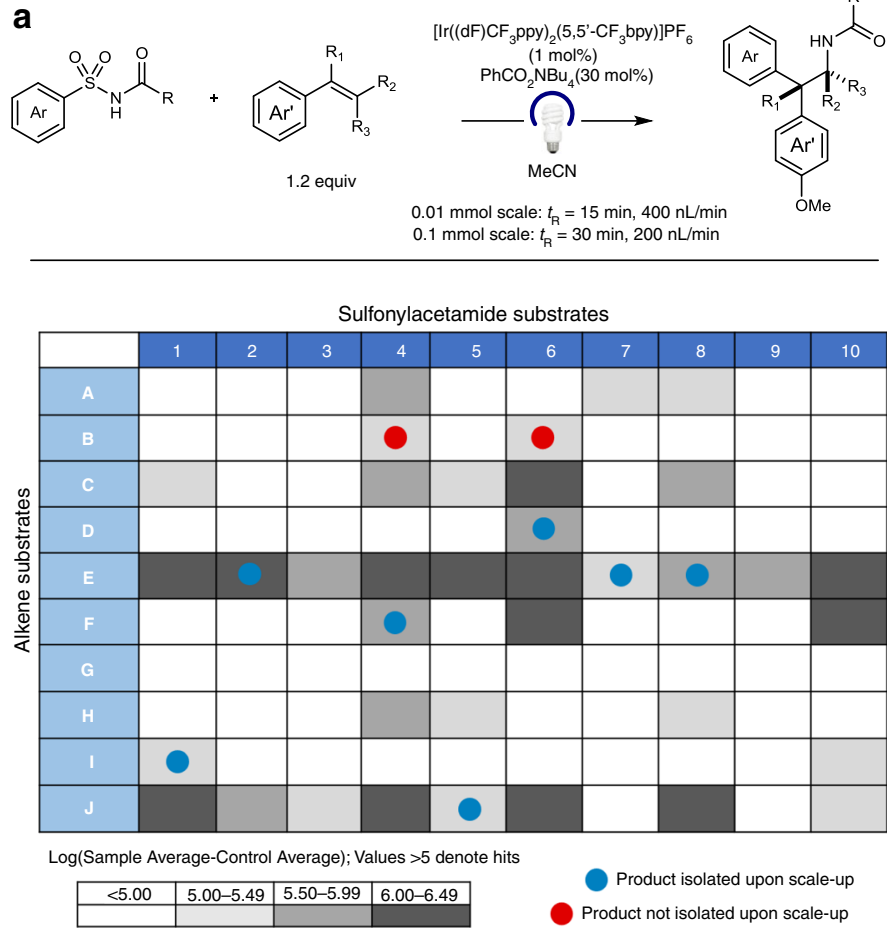

b

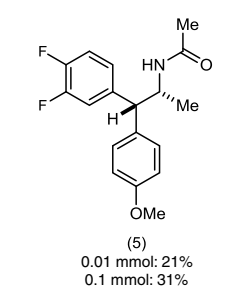

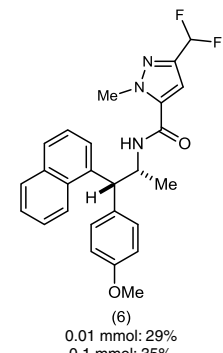

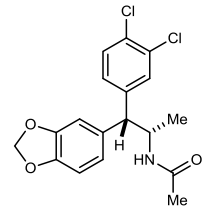

(7) 0.01 mmol: $18 \%$

$$
0.1 \mathrm{mmol}: 35 \%
$$

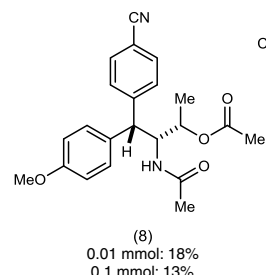

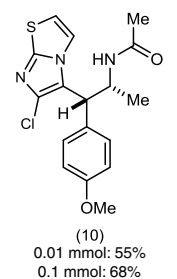

$0.01 \mathrm{mmol}: 18 \%$

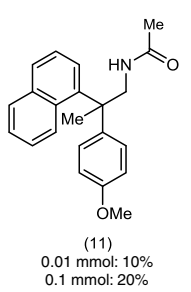

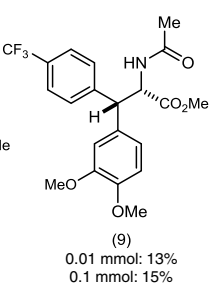

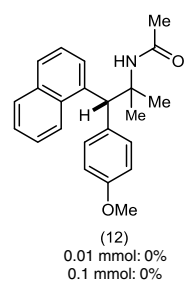

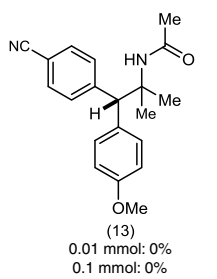

Fig. 3 Droplet microfluidics-enabled HTE reaction discovery on the picomole scale. a Heatmap of coupling 10 sulfonylacetamides with a matrix of 10 alkene substrates. Gray boxes denote potential hit/hit responses. Boxes containing red and blue circles indicate reactions that were performed on 0.01 $\mathrm{mmol}$ and $0.1 \mathrm{mmol}$ scale. b Product yields for millimole scale-up flow reactions. $\left[\operatorname{lr}\left((\mathrm{dF}) \mathrm{CF}_{3} \text { ppy }\right)_{2}\left(5,5^{\prime}-\mathrm{CF}_{3}\right.\right.$ bpy $\left.)\right] \mathrm{PF}_{6} 4,4^{\prime}$-di-tert-butyl-2, $2^{\prime}$-bipyridine-bis3,5-difluoro-2-[5-(trifluoromethyl)-2-phenylpyridine] iridium (III) hexafluorophosphate.

enhanced reactivity and broader alkene compatibility. Our indroplet reaction discovery screen has significantly expanded upon the scope of our reported alkene aminoarylation methodology to incorporate substrates of increased structural complexity, as well as enabled the elucidation of reactivity trends and structureactivity relationships to inform ongoing mechanistic studies.

Continuous flow scale-up validation. To validate our ESI-MS screen results, we selected nine of our droplet reactions to perform $0.01 \mathrm{mmol}$ scale-up in-flow and enable subsequent product isolation (Fig. 3). These reactions were carried out in the same reactor $(100 \mu \mathrm{m}$ i.d., 360 o.d.) and run in a continuous stream (non-droplet format) at a flow rate of $400 \mathrm{~nL} / \mathrm{min}$, providing a residence time of $15 \mathrm{~min}$. Upon irradiation, purification was performed using mass-directed HPLC methods. Of the nine reactions selected, seven of the hit reactions were successfully validated through product isolation. Product isolation was unsuccessful in the case of compounds 12 and 13 . Of the selected reactions, these yielded the weakest product MS signals in our original droplet reaction screen and had been categorized as potential hits. Control experiments suggest the formation of an unidentified byproduct that gives the same $\mathrm{m} / \mathrm{z}$ signal as the desired product (see Supplementary Information for details). Finally, we set out to demonstrate the translatability of our droplet screen results to a microscale flow reaction, in order to generate milligram scale quantities of material for discovery chemistry applications. Our $0.1 \mathrm{mmol}$ scale reactions were irradiated in a $100 \mu \mathrm{L}$ volume PFA reactor $\left(0.03^{\prime \prime}\right.$ i.d.) with a residence time of $30 \mathrm{~min}$ at a flow rate of $3 \mu \mathrm{L} / \mathrm{min}$. The same set of nine reactions were scaled up to yield isolation results that showed a strong correlation with that of the $0.01 \mathrm{mmol}$ scale flow reactions. These experiments highlight the utility of our droplet microfluidics platform for enabling reaction discovery in a high- throughput, material efficient, and data-rich manner. At the same time, this platform shows significant promise in its translatability and applicability towards discovery chemistry scale-up to facilitate library synthesis for initial biological studies.

Design of a reagent addition system. While the droplets used in the reactors shown in Figs. 2 and 3 contain nanoliters of reagents, the true reagent consumption was microliters that were deposited into the well plate. To fully realize the potential for reduced reagent consumption, we integrated automated reagent addition capabilities into a droplet reactor (Fig. 4a). By adding reagents (e.g., photocatalyst) directly to substrate-containing droplets, reagent consumption can be reduced to picomoles per reaction. As depicted in Fig. 4, preformed substrate-containing acetonitrile droplets were flowed from PFA tubing into a reagent addition device, through an addition region to receive photocatalyst, and exported to PFA tubing for irradiation and ESI-MS analysis (throughput of 0.3 samples/s) in one continuous system. Consistent volume addition $(<9 \%$ relative standard deviation for added reagent in two separate experiments; 48 droplets examined in each experiment) and low droplet-to-droplet carry-over were observed in preliminary experiments (Supplementary Fig. 8). This platform was subsequently utilized to perform and monitor indroplet photochemistry. ESI-MS analysis of aminoarylation droplets after photocatalyst addition and irradiation $(7 \mathrm{~min}$ residence time) showed consistent product formation across all droplet samples (Supplementary Fig. 9). Because these reactions are performed continuously, after the initial $7 \mathrm{~min}$ lag, reactions are analyzed at a rate limited by detection, i.e., 0.3 samples/s. These experiments provide proof-of-concept for the enhanced material efficiency of our developed platform, as droplet reactions were prepared and analyzed at $<1 \%$ sample usage relative to conventional $\mu \mathrm{L}$-scale screens while providing high-throughput in a 


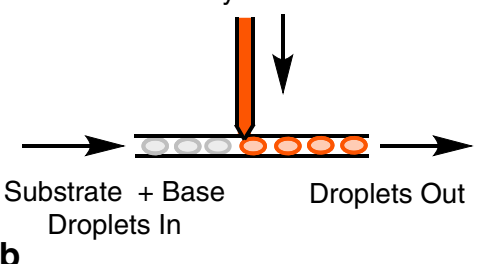

b
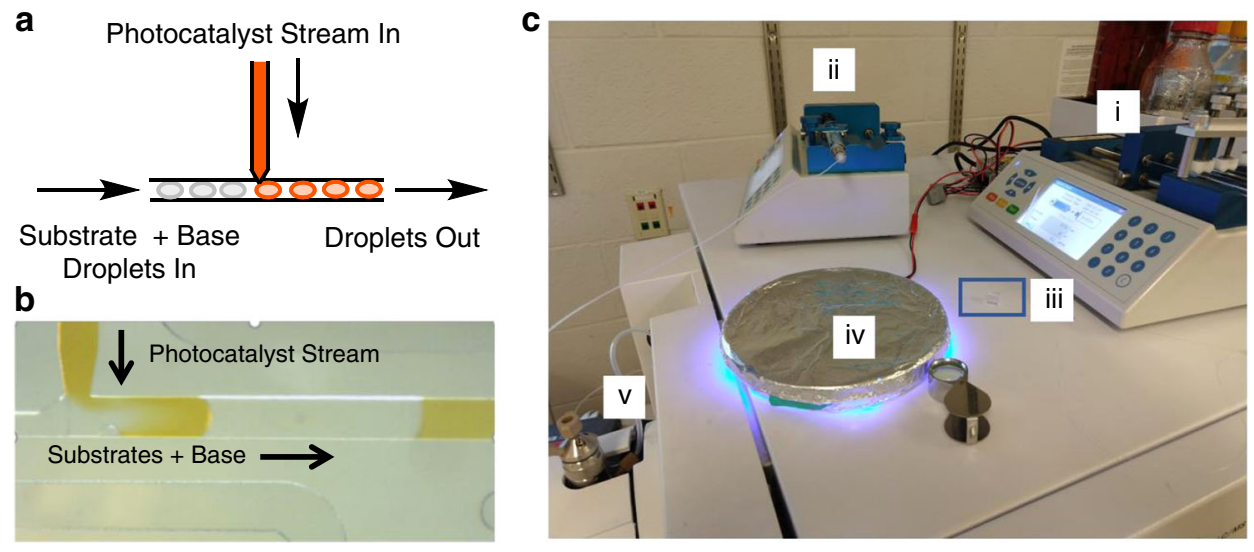

Fig. 4 Incorporation of a microfluidic chip for reagent addition. a Reagent addition device design. $\mathbf{b}$ Device in operation. Each incoming droplet from the left received a solution from the upper channel and moved right to export. c Full setup for in-droplet flow reaction screening. (i) Syringe pump driving both droplet flow and reagent flow into reagent addition chip, (ii) Syringe pump driving sheath flow, (iii, in blue box) Reagent addition device, (iv) Photoreactor chamber, (v) Sheath sprayer for ESI-MS analysis.

flow-based reaction. We envision the applicability of this system towards screening efforts in photocatalysis, as well as other nonphotochemical catalytic transformations, in which reagents are expensive or limited in supply.

\section{Discussion}

The droplet microfluidics system described in this study provides a high-throughput continuous flow platform for screening large volumes of photochemical reactions in a miniaturized fashion. The use of droplet microfluidics presents several significant advantages for performing in-flow reaction discovery. Our oscillatory in-droplet screening setup (Fig. 3) allows for the simultaneous irradiation of up to 100 picomole-scale reaction droplets, representing a marked improvement from state-of-theart systems that typically accommodate one sample/incubation period (Supplementary Table 1) ${ }^{19,32}$. Furthermore, this system enables in-droplet reaction discovery to rapidly generate highvolume compound libraries and provide access to greater magnitudes of chemical space. ESI-MS analysis of reaction droplets was performed at a throughput of 0.3 samples/s, which represents a marked improvement over other ESI-MS systems for the analysis of flow reactions ${ }^{20}$. Upon translating our picomole-scale droplet reactions to millimole scale flow conditions, we have also validated the successful flow scale-up of our droplet reactions to enable product isolation.

In comparison with plate-based screening approaches ${ }^{7,8,10}$, our system provides enhanced levels of material efficiency (500 pmol of material usage per reaction vs. 1-50 nmol) and high acquisition throughputs (Supplementary Table 1). While DESI-MS (desorption electrospray ionization-mass spectrometry) methods provide superior analytical throughputs (1-2 samples/s), they do not accommodate photochemical flow reactions, as reaction samples need to be analyzed in plate format. Compared to other similar screening platforms $s^{9,20,24}$, the platform reported here expands upon the utility of flow-based HTE systems beyond Suzuki cross-coupling and thermal reactions to enable screening of photochemical conditions, shows significantly enhanced material efficiency (500 pmol per reaction vs. $200-800 \mathrm{nmol}$ ) and increases analytical throughput over 10 -fold (0.3 samples/s vs. 0.02 samples/s).

Future studies will be targeted towards developing a microfluidic chip-based system to enable in-line droplet generation, reagent addition, incubation, and MS analysis. Automated data processing capabilities will be also be developed to accommodate the wealth of HTE data generated from larger screening campaigns. From an early discovery standpoint, this droplet microfluidics HTE platform will facilitate rapid photochemical reaction discovery in-flow for the expedited generation of compound libraries. We anticipate that droplet microfluidics-driven HTE will continue to expand and enhance the utility of photochemical transformations from the bench to the drug pipeline.

\section{Methods}

Procedure for trifluoromethylation in-droplet reactions. Photocatalyst ( $1 \mathrm{~mol} \%)$, pyridine $\mathrm{N}$-oxide (4 equiv.), and acetonitrile $(0.2 \mathrm{M})$ were added to a vial charged with a stir bar. The solution was sparged with a stream of nitrogen gas for $5 \mathrm{~min}$. Trifluoroacetic anhydride (4 equiv.) was subsequently added, and the solution was stirred for $10 \mathrm{~min}$ to facilitate the formation of the acylated species. Separate solutions of the substrate in acetonitrile $(0.2 \mathrm{M})$ were also prepared. $10 \mu \mathrm{L}$ of each solution were combined in a PCR tube to yield the final reaction mixture, which was then deposited into a 1536 multiwell plate for droplet generation. Droplets of $4 \mathrm{~nL}$ volume were formed from each mixture into reactor tubing $(100 \mu \mathrm{m}$ i.d., $360 \mu \mathrm{m}$ o.d.) and placed into a reactor dish (Supplementary Fig. 2) for irradiation. After $10 \mathrm{~min}$ of irradiation, droplet samples were characterized by ESI-MS analysis.

Representative procedure for Smiles-Truce rearrangement in-droplet reaction screen. To a flame dried 1-dram vial was added tetrabutylammonium benzoate $(30 \mathrm{~mol} \%)$, and $\left[\operatorname{Ir}\left(\mathrm{dF}\left(\mathrm{CF}_{3}\right) \text { ppy }\right)_{2}\left(5,5^{\prime} \mathrm{d}\left(\mathrm{CF}_{3}\right)\right.\right.$ bpy) $\mathrm{PF}_{6}$ photocatalyst $(1$ $\mathrm{mol} \%)$. The vial contents were then dissolved in anhydrous acetonitrile $(0.2 \mathrm{M})$. Finally, the alkene was added (1.2 equiv.). This solution was sparged under argon for $15 \mathrm{~min}$. Separate solutions of the substrate in acetonitrile $(0.2 \mathrm{M})$ were also prepared. For reactions formed directly from well-plates, $10 \mu \mathrm{L}$ of each solution were deposited into a well to form the final reaction mixture. Three droplets were made for every reaction condition, with the average droplet response reported. 10 droplets for every sulfonamide substrate were run in which no alkene reagent was present to generate an average control value. Following droplet generation, reactor tubing ( $100 \mu \mathrm{m}$ i.d., $360 \mu \mathrm{m}$ o.d.) containing droplets was wrapped around a $100 \times$ $50 \mathrm{~mm}$ glass recrystallization dish and placed on top of our 25 LED array light setup (Supplementary Fig. 4) for irradiation. Droplet reactions were run at a flow rate of $200 \mathrm{~nL} / \mathrm{min}$ in an oscillatory manner by programming a syringe pump to alternate between refill and infusion modes at $10 \mathrm{~min}$ intervals, yielding a total residence time of $1 \mathrm{~h}$. It is noteworthy that residence times were varied based on experiment. Following irradiation, droplet samples were characterized by ESI-MS analysis.

ESI-MS analysis of droplet samples. Tubing containing droplets was threaded through capillary electrophoresis (CE) ESI-MS sprayer until $\sim 0.5 \mathrm{~mm}$ was protruding. Sheath and droplet flows were driven by Fusion 400 syringe pumps. Droplets were flowed into the sheath sprayer (Supplementary Fig. 2) and merged with a dilution stream of 50:50 methanol:water w/0.5\% formic acid $(100 \mu \mathrm{L} / \mathrm{min}$ flow rate). ESI-MS analysis was performed on an Agilent 6410 triple quadrupole mass spectrometer. ESI potential was set to $2500 \mathrm{~V}$, nebulizer gas to $15 \mathrm{psi}$, and drying gas from MS source flowed at $10 \mathrm{~L} / \mathrm{min}$ at $325^{\circ} \mathrm{C}$. The mass spectrometer was set to scan from 75 to $750 \mathrm{~m} / z$ at $73 \mathrm{~ms}$ per scan. Droplet responses for any given $\mathrm{m} / z$ value were taken as the average of three consecutive data points from within each droplet's observed peak. 


\section{Data availability}

The data that support the findings of this study are available from the corresponding author upon reasonable request.

Received: 3 June 2020; Accepted: 4 November 2020;

Published online: 03 December 2020

\section{References}

1. Chow, S., Liver, S. \& Nelson, A. Streamlining bioactive molecular discovery through integration and automation. Nat. Rev. Chem. 2, 174-183 (2018).

2. Gromski, P. S., Henson, A. B., Granda, J. M. \& Cronin, L. How to explore chemical space using algorithms and automation. Nat. Rev. Chem. 3, 119-128 (2019).

3. Allen, C. L., Leitch, D. C., Anson, M. S. \& Zajac, M. A. The power and accessibility of high-throughput methods for catalysis research. Nat. Catal. 2, 2-4 (2019)

4. Milo, A. Democratizing synthesis by automation. Science 363, 122-123 (2019).

5. Krska, S. W., DiRocco, D. A., Dreher, S. D. \& Shevlin, M. The evolution of high-throughput chemical experimentation to address challenging problems in pharmaceutical synthesis. Acc. Chem. Res. 50, 2976-2985 (2017).

6. Schmink, J. R., Bellomo, A. \& Berritt, S. Scientist-led high-throughput experimentation (HTE) and its utility in academia and industry. Aldrichim. Acta 46, 71-80 (2017).

7. Santanilla, A. B. et al. Nanomole-scale high-throughput chemistry for the synthesis of complex molecules. Science 347, 443-448 (2015).

8. Gesmundo, N. J. et al. Nanomole synthesis and affinity ranking. Nature 557, 228-232 (2018).

9. Perera, D. et al. A platform for automated nanomole-scale screening and micromole-scale synthesis in flow. Science 359, 429-434 (2018).

10. Wleklinski, M. et al. High-throughput reaction screening using desorption electrospray ionization mass spectrometry. Chem. Sci. 9, 1647-1653 (2018).

11. McAtee, R. C., McClain, E. J. \& Stephenson, C. R. J. Illuminating photoredox catalysis. J. Trends Chem. 1, 111-125 (2019).

12. Cambié, D., Bottecchia, C., Straathof, N. J. W., Hessel, V. \& Noël, T. Applications of continuous-flow photochemistry in organic synthesis, material science, and water treatment. Chem. Rev. 116, 10276-10341 (2016).

13. Bédard, A. C. et al. Reconfigurable system for automated optimization of diverse chemical reactions. Science 361, 1220-1225 (2018).

14. Douglas, J. J., Sevrin, M. J., Cole, K. P. \& Stephenson, C. R. J. Visible light photoredox catalysis: applications and new disconnections in the synthesis of pharmaceutical agents. Org. Process Res. Dev. 20, 1148-1155 (2016).

15. DiRocco, D. A. et al. Late-stage functionalization of biologically active heterocycles through photoredox catalysis. Angew. Chem. Int. Ed. 53, 4802-4806 (2014).

16. Kuijpers, K. et al. A fully automated continuous-flow platform for fluorescence quenching studies and Stern-Volmer analysis. Angew. Chem. Int. Ed. 57, 11278-11282 (2018).

17. Pieber, B., Shalom, M., Antonietti, M., Seeberger, P. H. \& Gilmore, K. Continuous heterogeneous photocatalysis in serial micro-batch reactors. Angew. Chem. Int. Ed. 57, 9976-9979 (2018).

18. Hsieh, H.-W., Coley, C. W., Baumgartner, L. M., Jensen, K. F. \& Robinson, R. I. Photoredox iridium-nickel dual-catalyzed decarboxylative arylation crosscoupling: from batch to continuous flow via self-optimizing segmented flow reactor. Org. Process Res. Dev. 22, 542-550 (2018).

19. Hwang, Y. J. et al. A segmented flow platform for on-demand medicinal chemistry and compound synthesis in oscillating droplets. Chem. Commun. 53, 6649-6652 (2017).

20. Beulig, R. J. et al. A droplet-chip/mass spectrometry approach to study organic synthesis at nanoliter scale. Lab Chip 17, 1996-2002 (2017).

21. Shang, L., Cheng, Y. \& Zhao, Y. Emerging droplet microfluidics. Chem. Rev. 117, 7964-8040 (2017).

22. Tucker, J., Zhang, Y., Jamison, T. F. \& Stephenson, C. R. J. Visible photoredox catalysis in flow. Angew. Chem. Int. Ed. 51, 4144-4147 (2012).

23. Corcoran, E., McMullen, J. P., Lévesque, F., Wismer, M. K. \& Naber, J. R. Photon equivalents as a parameter for scaling photoredox reactions in flow: translation of a photocatalytic C-N cross-coupling from lab scale to multikilogram scale. Angew. Chem. Int. Ed. 59, 11964-11968 (2020).

24. Coley, C. W., Abolhasani, M., Lin, H. \& Jensen, K. F. Material-efficient microfluidic platform for exploratory studies of visible-light photoredox catalysis. Angew. Chem. Int. Ed. 56, 9847-9850 (2017).

25. Sun, S., Slaney, T. R. \& Kennedy, R. T. Label-free screening of enzyme inhibitors at femtomole scale using segmented flow electrospray ionization mass spectrometry. Anal. Chem. 84, 5794-5800 (2012).
26. Sun, S. \& Kennedy, R. T. Droplet electrospray ionization mass spectrometry for high throughput screening for enzyme inhibitors. Anal. Chem. 86, 9309-9314 (2014)

27. Diefenbach, X. W. et al. Enabling biocatalysis by high-throughput protein engineering using droplet microfluidics coupled to mass spectrometry. ACS Omega 3, 1498-1508 (2018).

28. Steyer, D. J. \& Kennedy, R. T. High-throughput nanospray electrospray ionization mass spectrometry analysis of microfluidic droplet samples. Anal. Chem. 91, 6645-6651 (2019).

29. Beatty, J. W., Douglas, J. J., Cole, K. P. \& Stephenson, C. R. J. A scalable and operationally simple radical trifluoromethylation. Nat. Commun. 6, 7919-7925 (2015)

30. Pfizer. Pfizer commercial compound library link https://www.sigmaaldrich. com/life-science/cell-biology/bioactive-small-molecules/pfizer.html.

31. Monos, T. M., McAtee, R. C. \& Stephenson, C. R. J. Arylsulfonylacetamides as bifunctional reagents for alkene aminoarylation. Science 361, 1369-1373 (2018).

32. Abolhasani, M., Bruno, N. C. \& Jensen, K. F. Oscillatory three-phase flow reactor for studies of bi-phasic catalytic reactions. Chem. Commun. 51, 8916-8919 (2015)

\section{Acknowledgements}

We would like to thank Dr. Joseph Tucker for providing compounds from Pfizer's commercial compound library. We acknowledge the financial support for this research from the NSF (CBET-1604087 and CHE-1900266) and the University of Michigan. This manuscript was developed with the support of the American Chemical Society Green Chemistry Institute Pharmaceutical Roundtable (https://www.acs.org/content/acs/en/ greenchemistry/industry-business/pharmaceutical.html). The ACS GCI is a not-forprofit organization whose mission is to catalyze and enable the implementation of green and sustainable chemistry throughout the global chemistry enterprise. The ACS GCI Pharmaceutical Roundtable is composed of pharmaceutical and biotechnology companies and was established to encourage innovation while catalyzing the integration of green chemistry and green engineering in the pharmaceutical industry. The activities of the Roundtable reflect its members' shared belief that the pursuit of green chemistry and engineering is imperative for business and environmental sustainability. This material is based on work supported by an NSF Graduate Research Fellowship (Grant DGE 1256260) (A.C.S.).

\section{Author contributions}

A.C.S., D.J.S., A.R.A., and E.P. performed the experiments. A.C.S., D.J.S., C.R.J.S., and R.T.K. designed the experiments. A.C.S., D.J.S., C.R.J.S., and R.T.K. wrote the manuscript.

\section{Competing interests}

The authors declare no competing interests.

\section{Additional information}

Supplementary information is available for this paper at https://doi.org/10.1038/s41467 020-19926-z.

Correspondence and requests for materials should be addressed to R.T.K. or C.R.J.S

Peer review information Nature Communications thanks the anonymous reviewer(s) for their contribution to the peer review of this work.

Reprints and permission information is available at http://www.nature.com/reprints

Publisher's note Springer Nature remains neutral with regard to jurisdictional claims in published maps and institutional affiliations.

pen Access This article is licensed under a Creative Commons Attribution 4.0 International License, which permits use, sharing, adaptation, distribution and reproduction in any medium or format, as long as you give appropriate credit to the original author(s) and the source, provide a link to the Creative Commons license, and indicate if changes were made. The images or other third party material in this article are included in the article's Creative Commons license, unless indicated otherwise in a credit line to the material. If material is not included in the article's Creative Commons license and your intended use is not permitted by statutory regulation or exceeds the permitted use, you will need to obtain permission directly from the copyright holder. To view a copy of this license, visit http://creativecommons.org/ licenses/by/4.0/

(c) The Author(s) 2020 